\title{
Quantitative coherent Raman scattering microscopy for bioimaging
}

\author{
Paola Borri \\ Cardiff University School of Biosciences, Sir Martin Evans Building, Museum Avenue, CF10 3AX Cardiff, United Kingdom \\ email: borrip@cf.ac.uk
}

Optical microscopy is an indispensable tool that is driving progress in cell biology and is still the only practical means of obtaining spatial and temporal resolution within living cells and tissues. Coherent Raman Scattering (CRS) microscopy has attracted increasing attention as a powerful multiphoton microscopy technique which overcomes the need for fluorescent labelling and yet retains biomolecular specificity and intrinsic 3D resolution [1]. Over the past 15 years, our laboratory has developed and demonstrated a range of label-free CRS microscope set-ups featuring innovative excitation/detection schemes.

Our second-generation coherent anti-Stokes Raman scattering (CARS) instrument is based on a single $5 \mathrm{fs}$ Ti:Sa laser source capable of exciting a wide vibrational range (from $1000 \mathrm{~cm}^{-1}$ to $3500 \mathrm{~cm}^{-1}$ ) which allows us to perform hyperspectral microscopy, whereby a vibrational spectrum is obtained for each spatial voxel [2]. This in turn has led to the development of quantitative chemical imaging algorithms to represent the hyperspectral dataset as a superposition of Raman spectra and concentration maps of individual chemical components (e.g. proteins, lipids, DNA) in physically meaningful units [3].

With this technique, we have measured the 3D spatial distribution of lipid droplets in live mouse oocytes and early embryos [4] and elucidated the link between this distribution and the use of lipids in the egg metabolism (generation of ATP by mitochondrial oxidation of fatty-acids) [5]. We have also measured the lipid uptake in living human adipose-derived stem cells differentiating into pre-adipocytes over 9 days, observing a heterogeneous behaviour which is droplet-size dependent, time dependent, and lipid dependent [6]. Furthermore, we have quantitatively measured volumetric concentrations (and in turn dry masses) of lipids, proteins and DNA in 3D during cell division [7]. In addition, we have shown that CARS can be used to visualise single non-fluorescing nanodiamonds in cells for the first time [8]. Recently, we have applied the technique to visualise lipid partitioning in single planar membranes bilayers exhibiting liquid ordered (Lo) and disordered (Ld) domains. We find that that domains are consistently resolved, both chemically and spatially, in a completely label-free manner [9]. It should be stressed that imaging single membrane bilayers is pushing the detection sensitivity of CRS to its limit.

In terms of further instrumentation developments, we have implemented a high-speed high-throughput platform for drug screens, exploiting Bessel beam excitation and sparse sampling [10]. We have also developed a new interferometric CRS set-up, which offers background-free chemically-specific image contrast, shot-noise limited detection, and phase sensitivity, enabling topographic imaging of interfaces [11]. The technique features heterodyne detection of CARS in epi-geometry, as well as multi-modal acquisition of stimulated Raman scattering (SRS) and forward-emitted CARS intensity in the same instrument.

I will present our latest progress with these techniques and their applications to bio-imaging.

\section{References}

[1] A. Zumbusch, W. Langbein, P. Borri, Progress in Lipid Research 52, 615 (2013).

[2] I. Pope et al., Optics Express 21, 7096 (2013).

[3] F. Masia et al., Anal. Chem. 85, 10820 (2013).

[4] J. Bradley et al., Development 143, 2238 (2016).

[5] J. Bradley et al. J. of Cell Science 132, jcs228999 (2019).

[6] C. Di Napoli et al., Anal. Chem. 88, 3677-3685 (2016).

[7] A. Karuna et al., Anal. Chem. 91, 2813 (2019).

[8] I. Pope et al., Nat. Nanotechnol. 9, 940 (2014).

[9] A. Nahmad-Rohen et al., Anal. Chem. 92, 14657 (2020).

[10] F. Masia et al., Anal. Chem. 90, 3775 (2018).

[11] W. Langbein et al., APL Photonics 3, 092402 (2018). 